\section{Distribution of non-myelinating Schwann cells and their associations with leukocytes in mouse spleen revealed by immunofluorescence staining}

\author{
Bin Ma, ${ }^{1}$ Changfu Yin, ${ }^{2}$ Dailun $\mathrm{Hu},{ }^{2}$ \\ Mark Newman, ${ }^{1}$ Philip K. Nicholls, ${ }^{1}$ \\ Zhanjun Wu, ${ }^{3}$ Wayne K. Greene, ${ }^{1}$ \\ Zhongli Shi
}

${ }^{1}$ School of Veterinary and Life Sciences, Murdoch University, Murdoch, WA, Australia

${ }^{2}$ Clinical College, Hebei Medical University, Shijiazhuang, Hebei, China ${ }^{3}$ Institute of Cereal and Oil Crop, Hebei Academy of Agriculture and Forestry Sciences, Shijiazhuang, Hebei, China

\section{Abstract}

The nervous system and the immune system communicate extensively with each other in order to maintain homeostasis and to regulate the immune response. The peripheral nervous system (PNS) communicates specifically with the immune system according to local interactions, including the "hardwiring" of sympathetic/parasympathetic (efferent) and sensory nerves (afferent) to lymphoid tissue and organs. To reveal this type of bidirectional neuroimmune interaction at the microscopic level, we used immunofluorescent staining of glial fibrillary acidic protein (GFAP) coupled with confocal microscopy/3D reconstruction to reveal the distribution of nonmyelinating Schwann cells (NMSCs) and their interactions with immune cells inside mouse spleen. Our results demonstrate i) the presence of an extensive network of NMSC processes in all splenic compartments including the splenic nodules, periarteriolar lymphoid sheath (PALS), marginal zone, trabecula, and red pulp; ii) the close association of NMSC processes with blood vessels (including central arteries and their branches, marginal sinuses, penicillar arterioles and splenic sinuses); iii) the close "synapse-like" interaction/association of NMSC processes with various subsets of dendritic cells (DCs; e.g., $\mathrm{CD}^{+} \mathrm{CD} 11 \mathrm{c}^{+}$ $\mathrm{DCs}, \mathrm{B} 220^{+} \mathrm{CD} 11 \mathrm{c}^{+} \mathrm{DCs}$, and $\mathrm{F} 4 / 80^{+}$ CD11 $\left.\mathrm{c}^{+} \mathrm{DCs}\right)$, macrophages $\left(\mathrm{F} 4 / 80^{+}\right)$, and lymphocytes (B cells, $\mathrm{CD}^{+} \mathrm{T}$ helper cells). Our novel findings concerning the distribution of NMSCs and NMSC-leukocytes interactions inside mouse spleen should improve our understanding of the mechanisms through which the PNS affects cellu- lar- and humoral-mediated immune responses in a variety of health conditions and infectious/non-infectious diseases.

\section{Introduction}

The nervous system and the immune system communicate extensively with each other in order to maintain homeostasis and to protect the host against infectious and non-infectious diseases. ${ }^{1-5}$ The central nervous system (CNS) regulates the immune system by the secretion of hormones from the pituitary and other endocrine organs, ${ }^{6-8}$ whereas the peripheral nervous system (PNS) communicates specifically with the immune system according to local interactions, including the "hardwiring" of sympathetic/parasympathetic (efferent) and sensory nerves (afferent) to lymphoid organs. ${ }^{1,9,10}$ In addition, neurotransmitters such as acetylcholine, norepinephrine, vasoactive intestinal peptide, substance $\mathrm{P}$, and histamine released from nerve terminals or even from the immune cells can also modulate immune activity under a variety of conditions. . $^{11,12}$

The spleen acts as the primary filter for blood-borne pathogens/antigens and as a critical organ that is responsible for iron metabolism and erythrocyte homeostasis. In addition, the spleen, as a well-organized lymphoid organ, has other important immune and hematopoietic functions. ${ }^{13,14}$ Recent studies have demonstrated the interactions of PNS with spleen under healthy conditions and in diseases. ${ }^{9,15,16}$ Neural input into the spleen by both sympathetic and sensory nerve fibers has been demonstrated in many studies and across a wide variety of species including human and mouse. ${ }^{9,10,15-20}$ These splenic nerve fibers also have direct close associations with immune cells inside the spleen and play essential roles in the regulation of immunity. ${ }^{9,10,15,19}$ To gain a better understanding of the nerve-immune cell interactions in the spleen, the types of nerve fibers that might be present in the spleen need to be identified. The CNS has relatively few unmyelinated nerve fibers and, thus, benefits from the advantages of myelination, including faster conduction velocities, lower energy consumption, and higher stability of pointto-point connectivity. ${ }^{21}$ On the contrary, in the PNS, many nerve fibers or parts of nerve fibers (approximately 80\%) are nonmyelinated. These nerve fibers include the Group C nerve fibers (carrying sensory information), the postganglionic sympathetic fibers, some of the preganglionic sympathetic/parasympathetic fibers, and the motor nerve terminals at neuromuscular junctions. $^{22}$ The Schwann cells of the
Correspondence: Zhongli Shi, Clinical College, Hebei Medical University, 309 Jianhuanan Street, Shijiazhuang, Hebei 050031, China.

Tel. +86.311.86265948 - Fax: +86.311.86265911. E-mail: shizhongli@hebmu.edu.cn

Key words: Non-myelinating Schwann cells; lymphocyte; dendritic cell; spleen; immunofluorescence staining.

Contributions: ZS, WKG, PKN, BM, conceived the study, designed the experiments, and wrote the paper; CY, DH, MN, ZW, BM, performed experiments and analyzed the data. All authors discussed the results, provided comments, and reviewed the manuscript.

Conflict of interest: None of the authors has a conflict of interest to declare.

Acknowledgements: The authors would like to thank Wayne Rasband for the ImageJ program.

Received for publication: 26 December 2017. Accepted for publication: 9 March 2017.

This work is licensed under a Creative Commons Attribution-NonCommercial 4.0 International License (CC BY-NC 4.0).

CC Copyright B. Ma et al., 2018

Licensee PAGEPress, Italy

European Journal of Histochemistry 2018; 62:2890 doi:10.4081/ejh.2018.2890

unmyelinated nerve fibers, namely the nonmyelinating Schwann cells (NMSCs), can act as the "first responders" to injury or disease in their neighborhoods. ${ }^{22}$ The Remak fibers (usually in the range of $0.5-1.5 \mu \mathrm{m}$ in diameter), whose Schwann cells form the primary populations of NMSCs, have small axons that can extend longitudinally for 50 $100 \mu \mathrm{m} .{ }^{23}$ In addition to being essential in neural regulation and repair, Schwann cells can act as immunocompetent cells and also play essential roles in immunomodulation. ${ }^{24-26}$ For example, Schwann cells can induce an immune response via patternrecognition receptors and trigger the $\mathrm{T}$ cell response via antigen presentation. ${ }^{24-25}$ Moreover, Schwann cells interact with lymphocytes, dendritic cells (DCs), and macrophages under healthy conditions and in diseases. ${ }^{27-29}$

Despite the studies mentioned above, the data on the innervation and nerveimmune interaction of spleen remain limited. In the present study, by using immunofluorescent staining and confocal microscopy/three-dimensional (3D) reconstruction, we have investigated the distribution of NMSCs and NMSC-immune cell interactions in situ in the mouse spleen in 
order to improve our understanding of the microanatomical basis of the interaction of PNS and secondary lymphoid tissue/organs, including spleen and lymph node.

\section{Materials and Methods}

\section{Animals}

C57BL/6 female mice (8-12-week-old) were purchased from the Animal Resources Centre (Perth, Australia). All animal experiments were carried out in accordance with the Australian code for the care and use of animals for scientific purposes at Murdoch University, Perth, Australia, with local animal ethics committee approval. In total, eight mice were used for the study.

\section{Section preparation}

Microscope slides $(27 \mathrm{~mm} \times 75 \mathrm{~mm})$ were obtained from Thermo Fisher Scientific (Scoresby, Australia). After a brief wash with $70 \%$ ethanol, the coverslips were coated with $0.01 \%$ poly-L-lysine solution (PLL; Sigma-Aldrich, St. Louis, MO, USA) for 10 min followed by air-drying overnight at room temperature.

Mice were killed by carbon dioxide followed by cervical dislocation. Their spleens were then quickly removed, embedded in Tissue-Tek ${ }^{\circledR} \quad$ O.C.T. COMPOUND (ProSciTech, Kinwan, Australia), and snapfrozen in liquid nitrogen. Cryosections (20 $\mu \mathrm{m})$ were prepared by using a Leica CM1850 UV Cryostat (Leica Biosystems, Nussloch, Germany) and mounted on the above-mentioned PLL-treated microscope slide.

\section{Antibodies}

Specificities and sources of antibodies are described in Table 1.

\section{Immunofluorescent staining}

Sections were fixed in methanol at $4^{\circ} \mathrm{C}$ for $3 \mathrm{~min}$, and then in acetone at $4^{\circ} \mathrm{C}$ for $3 \mathrm{~min}$. All the following steps were performed at room temperature. The sections were airdried for $1 \mathrm{~h}$ and then rehydrated for $10 \mathrm{~min}$ in phosphate-buffered saline (PBS). All washes $(3 \times 10 \mathrm{~min})$ between stages were performed in PBS. After the sections had been permeabilized with $0.2 \%$ Triton X-100 (Sigma-Aldrich) in PBS for $5 \mathrm{~min}$, potential non-specific binding sites were blocked with antibody dilution buffer $[2 \%$ goat serum (Sigma-Aldrich) and 1\% IgG-free bovine serum albumin (Sigma-Aldrich) in PBS] for $20 \mathrm{~min}$ at room temperature. Sections were then incubated with primary antibodies overnight at $4^{\circ} \mathrm{C}$. After being washed, the sections were then incubated with secondary antibodies for 1 hour at room temperature. Following the final washing step, sections were mounted with Fluorescence Mounting Medium (Dako, North Sydney, Australia).

For immunostaining with two rabbit antibodies [anti-glial fibrillary acidic protein (GFAP) and anti-p75 neurotrophin receptor $\left.\left(\mathrm{p} 75^{\mathrm{NTR}}\right)\right]$ on the same section, a sequential immunostaining approach ${ }^{30}$ was used [anti-GFAP+goat anti-rabbit IgG DyLight $488+$ rabbit IgG (Sigma) + (mixture of anti-p $75^{\mathrm{NTR}}+$ goat anti-rabbit $\mathrm{IgG}$ Alexa 555; incubated half-hour before application)].

\section{Confocal microscopy}

Confocal microscopy was performed with a Nikon Instruments C2 Plus Confocal
Microscope (Nikon Instruments, Melville, NY, USA) equipped with three lasers (excitation wavelength at $488 \mathrm{~nm}, 561 \mathrm{~nm}$ and $633 \mathrm{~nm}$ ). A Plan Apochromat $\lambda$ 20x objective lens and a Plan Apochromat $\lambda$ 40x objective lens were used for the imaging. For some micrographs, Tile Scan was performed to scan a large area of the spleen. After the acquisition, the images were adjusted and analyzed by using NISElements Advanced Research (AR) of the confocal system. Maximal intensity projection of a Z-Stack was performed by using the "Maximal intensity projection" function of the NIS-Elements AR program. The images obtained were then exported as TIFF files and further processed (adjustment of brightness and contrast)/edited (cropping and labelling) in Jasc Paint Shop Pro 9 (Corel Corporation, Ottawa, Canada).

\section{Colocalization analysis}

Colocalization analysis was performed by using ImageJ $1.51 \mathrm{~h}$ (https://imagej. net/Fiji/Downloads). Two images from blue and red channels were transformed into two grayscale images, and the colocalization was performed by using the "Colocalization" function in the "Analyze" menu. The colocalized structures were marked in white in the final images.

\section{Results}

In our previous study, ${ }^{29}$ we have applied GFAP successfully as a reliable marker for NMSCs inside the lymph node. We have used this antibody for immunofluorescent staining on a variety of tissues including brain, lung, trachea, skin, and intestine and observed brightly stained cells with astro-

Table 1. Specificities and sources of primary and secondary antibodies.

\begin{tabular}{|c|c|c|c|c|}
\hline Target & Conjugate & Species and isotype & Cells labelled & Company \\
\hline CD11c & & Armenian hamster monoclonal IgG & Dendritic cells & STEMCELL Technologies (Tullamarine, Australia) \\
\hline $\mathrm{B} 220$ (CD45R) & & Rat monoclonal IgG & B cell & Australian Biosearch (Karrinyup, Australia) \\
\hline CD31 & & Rat monoclonal IgG & Blood vessel endothelial cell & Australian Biosearch \\
\hline CD4 & & Rat monoclonal IgG & Thelper cell & Australian Biosearch \\
\hline $\mathrm{F} 4 / 80$ & & Rat monoclonal IgG & Red pulp macrophages & Australian Biosearch \\
\hline PGP9.5 & & Chicken polyclonal & Neuronal marker & Abcam Australia \\
\hline Neurofilament heavy polypeptide(H) & & Chicken polyclonal & Neuronal marker & Abcam Australia \\
\hline Glial fibrillary acidic protein (GFAP) & & Rabbit polyclonal & NMSCs & DAKO (North Sydney, Australia) \\
\hline P75NTR & & Rabbit polyclonal & NMSCs & Australian Biosearch \\
\hline Rabbit IgG H\&L & DyLight@ 488 & Goat polyclonal $\mathrm{F}\left(\mathrm{ab}^{\prime}\right) 2$ & & Abcam Australia (Melbourne, Australia) \\
\hline Armenian Hamster IgG H\&L & Alexa Fluor@ 647 & Goat polyclonal & & Abcam Australia \\
\hline Rat IgG H\&L & Alexa Fluor@ 555 & Goat polyclonal & & Abcam Australia \\
\hline Rabbit IgG H\&L & Alexa Fluor@ 555 & Goat polyclonal & & Abcam Australia \\
\hline Chicken IgG & Alexa Fluor® 555 & Goat polyclonal & & Abcam Australia \\
\hline
\end{tabular}


cyte morphology that was expected for the morphology and distribution for NMSCs in these tissues (data not shown). Close associations of GFAP and NMSC marker (such as p75 ${ }^{\text {NTR; }}$ Supplementary Figure 1a)/neuronal markers (protein gene product 9.5
(PGP9.5) and neurofilament; Supplementary Figure $1 b, c)$ revealed by co-staining/colocalization analysis has also demonstrated that GFAP is a reliable marker for NMSCs associated with Remak fibers. To understand the spatial relationship between
NMSC and blood vessels/DCs, tripleimmunolabelling with antibodies against GFAP, CD31 (a blood vessel endothelial cell marker), and CD11c was performed on $20-\mu \mathrm{m}$ cryosections from $\mathrm{C} 57 \mathrm{BL} / 6$ mouse spleen. The results are shown in

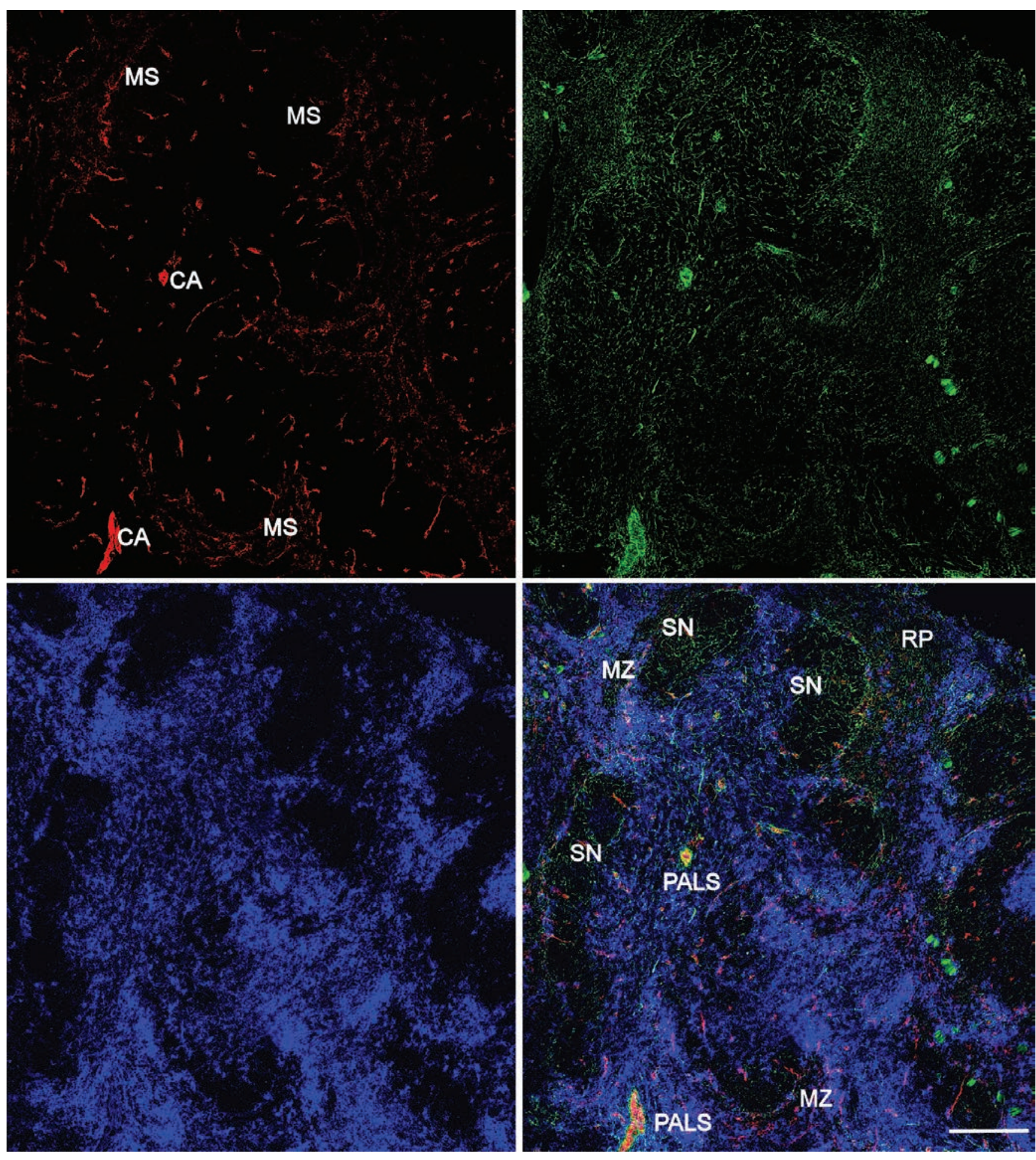

Figure 1. Distribution of NMSCs, blood vessels, and DCs in the C57BL/6 mouse spleen. Antibodies against GFAP (green), CD31 (red), and CD11c (blue) label mainly NMSCs, blood vessels, and DCs, respectively. The lower right panel is the merged image of the three immunolabellings.MS, marginal sinus; CA, central artery; MZ, marginal zone; SN, splenic nodule; PALS, periarteriolar lymphoid sheath; RP, red pulp. Objective lens: 20x; scanning mode: tile scan. Scale bar: $200 \mu \mathrm{m}$. 
Figures 1 and 2.

In Figure 1, Tile Scan (with a 20x objective lens) was utilized to reveal the distribution of NMSCs, blood vessels, and DCs in the white pulp of mouse spleen. An extensive meshwork of GFAP $^{+}$NMSC processes was seen throughout the spleen nodule, periarteriolar lymphoid sheath (PALS), and marginal zones. High-resolution confocal imaging by using a $40 \mathrm{x}$ objective lens was performed to reveal the distribution of
NMSCs, blood vessels, and DCs in each compartment of mouse spleen (Figure 2). In the PALS (Figure 2a), an extensive network of NMSC processes was observed, and these processes had close associations with the central artery and its branches, indicating the neuronal control of the blood flow. Around the central artery, DCs formed clusters, with many of them having close associations with NMSC processes (Figure 2A). The spleen nodule (Figure 2B) contained fewer extensive NMSC processes compared with the PALS (Figure 2A). In the marginal zone, the NMSC processes exhibited close associations with the marginal sinus and marginal zone DCs. The spleen red pulp (Figure 2C) possessed an extensive network of NMSC processes, which were closely associated with sheathed capillaries and splenic sinuses. In addition, many red pulp DCs interacted closely with NMSC processes.

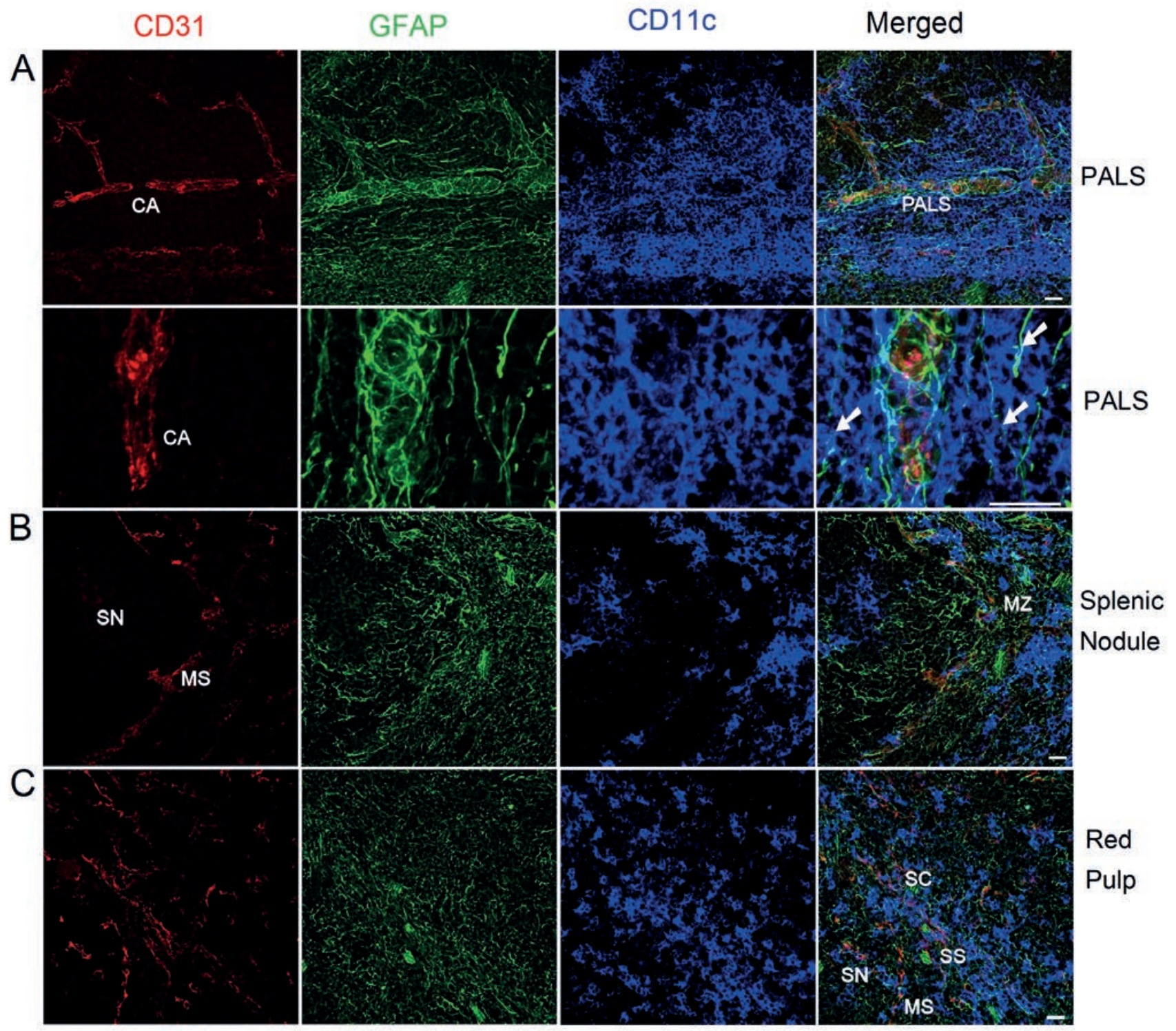

Figure 2. Distribution of NMSCs, blood vessels, and DCs in the PALS (A), splenic nodule (B), and red pulp (C) of C57BL/6 mouse spleen. Antibodies against GFAP (green), CD31 (red), and CD11c (blue) label mainly NMSCs, blood vessels, and DCs, respectively. A) The images in the second row are high-resolution views of another maximal intensity projection of the Z-stack shown in the first row; white arrows show the close interactions of NMSC processes and DCs in PALS. CA, central artery; PALS, periarteriolar lymphoid sheath; SN, splenic nodule; MS, marginal sinus; MZ, marginal zone; SS, splenic sinus; SC, splenic cord. Objective lens: 40x. Each image is a maximal intensity projection of a Z-stack. Stack size: $6.0 \mu \mathrm{m}$; optical slice interval: $0.50 \mu \mathrm{m}$. Scale bar: $20 \mu \mathrm{m}$. 
To reveal the distribution of NMSCs and their interactions with lymphocytes and DCs in mouse spleen, triple-immunolabelling was performed on $20-\mu \mathrm{m}$ cryosections of C57BL/6 mouse spleen. The distribution of NMSCs, B cells (revealed by antiB220 staining), and DCs is shown in Figures 3 and 4.
In Figure 3, Tile Scan (with 20x objective lens) was utilized to reveal the distribution of NMSCs, B cells, and DCs in red pulp and white pulp of mouse spleen. We observed that the $\mathrm{B}$ cell region had a less extensive network of NMSC processes compared with PALS and red pulp. Highresolution confocal imaging by using a 40x objective lens was performed to reveal the distribution of NMSCs, B cell, and DCs in PALS (Figure 4 A,C) and red pulp (Figure 4 B,D) of mouse spleen. In the PALS, an extensive network of NMSC processes was observed, and these processes had close associations with $\mathrm{B} 220^{+} \mathrm{B}$ cells and, B220-

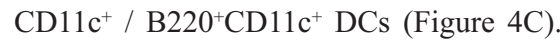
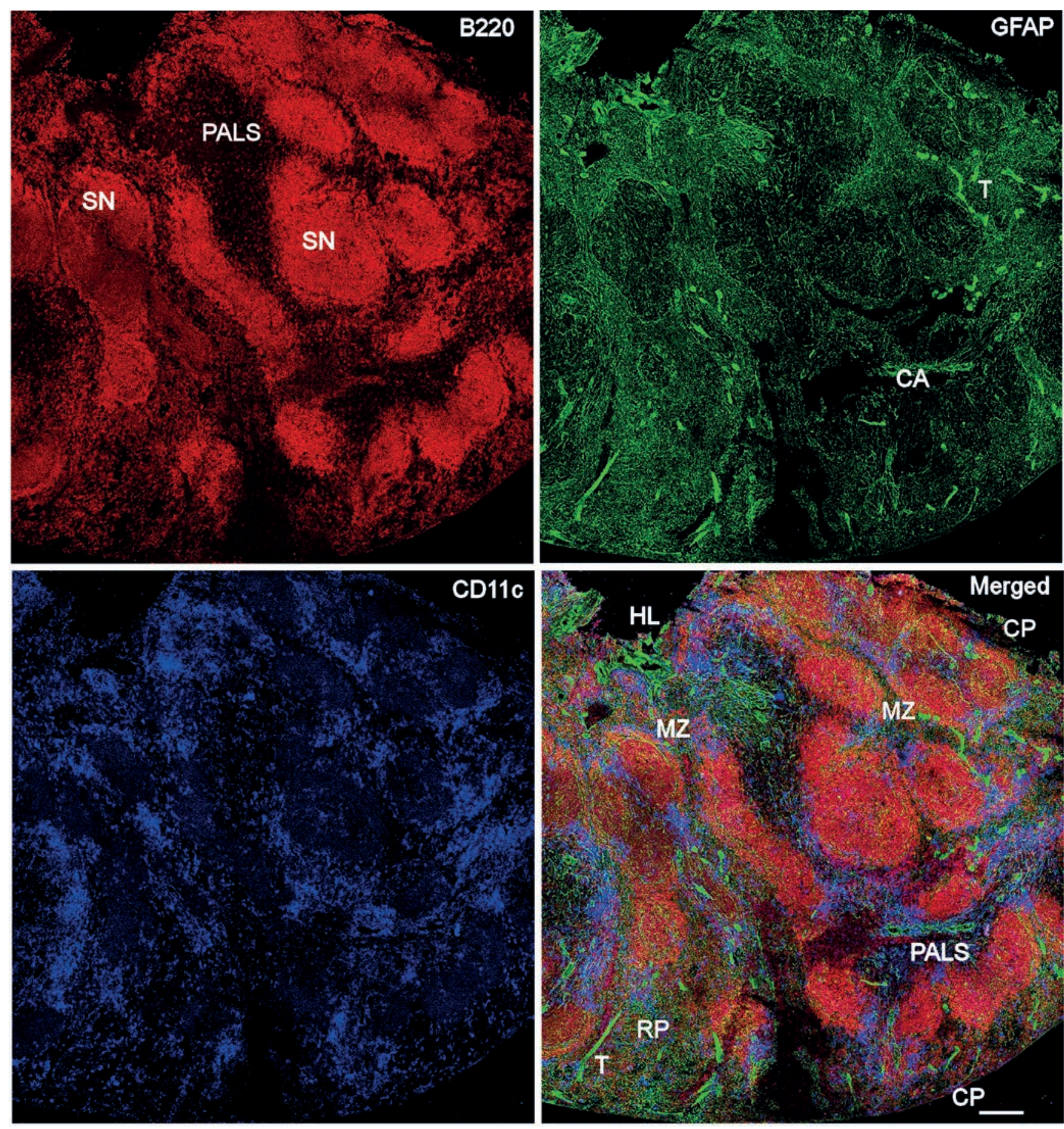

Figure 3. Distribution of NMSCs, B cells, and DCs in the C57BL/6 mouse spleen. Antibodies against GFAP (green), B220 (red), and CD11c (blue) label mainly NMSCs, B cells, and DCs, respectively. The lower right panel is the merged image of the three immunolabellings. CA, central artery; SN, splenic nodule; PALS, periarteriolar lymphoid sheath; CP, capsule; RP, red pulp; T, trabecula; HL, hilum. Objective lens: 20x; scanning mode: tile scan. Scale bar: $200 \mu \mathrm{m}$. 
This kind of interaction was also detected in splenic red pulp (Figure 4D).

We then checked the distribution of NMSCs, T helper cell (revealed by antiCD4 staining), and DCs, and the results are shown in in Figure 5. Few DCs were present in the splenic nodule (Figure 5A). In the splenic marginal zone, NMSCs processes were closely associated with
$\mathrm{CD}^{+} \mathrm{T}$ helper cells and CD4-CD11 ${ }^{+} /$ $\mathrm{CD}^{+}{ }^{+} \mathrm{CD} 11 \mathrm{c}^{+}$DCs (Figure 5A). The PALS contained an extensive network of NMSC processes that had close associations with a central artery (Figure 5B). Additionally, NMSCs processes exhibited close apposition to $\mathrm{CD}^{+} \mathrm{T}$ helper cells and $\mathrm{CD}^{-}$

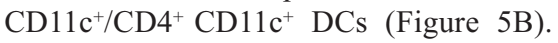
We also observed these kinds of interac- tion in spleen red pulp (Figure 5C).

As is known, the macrophage response can be triggered, maintained, and terminated by a two-way communication of macrophages and Schwann cells. In the present study, we investigated the crosstalk of NMSCs and a subset of splenic macrophage-F4/80 macrophages, and the results are shown in Figure 6. We observed an
B220
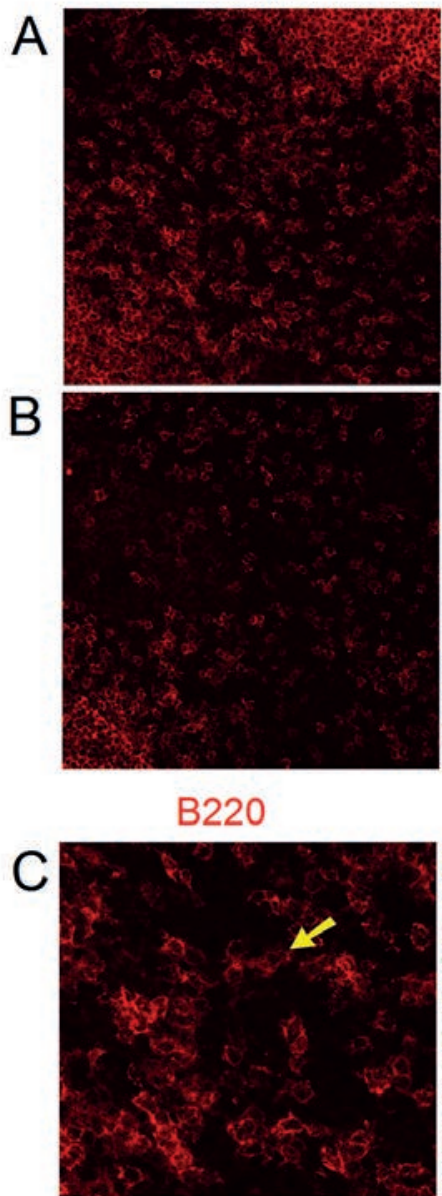

D

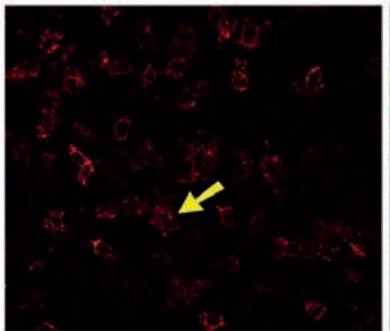

GFAP
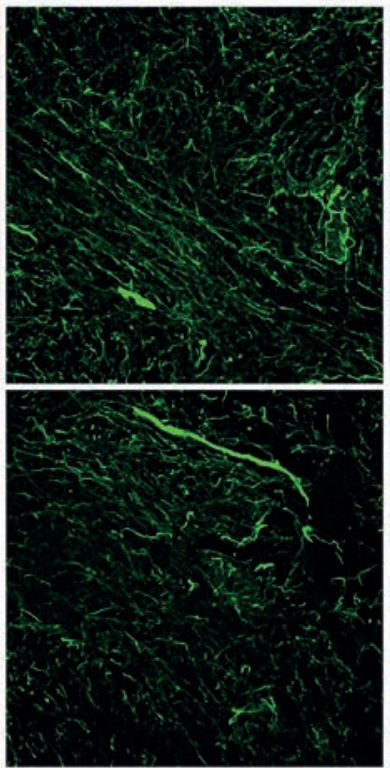

B220+GFAP
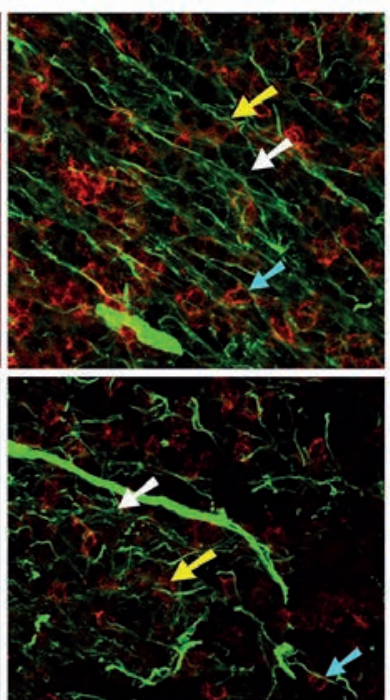

CD11c

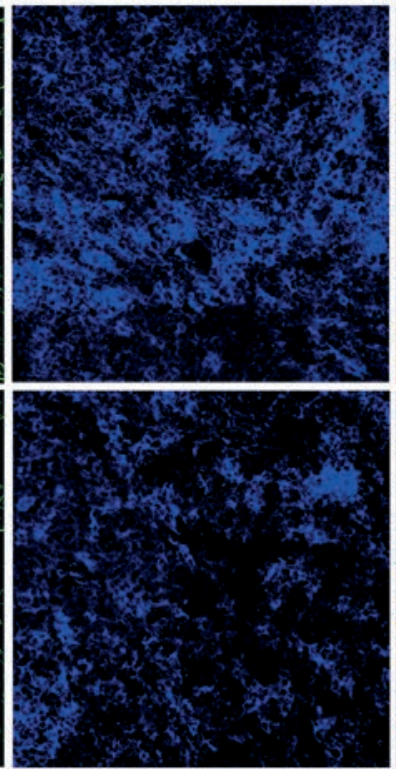

CD11c+ GFAP
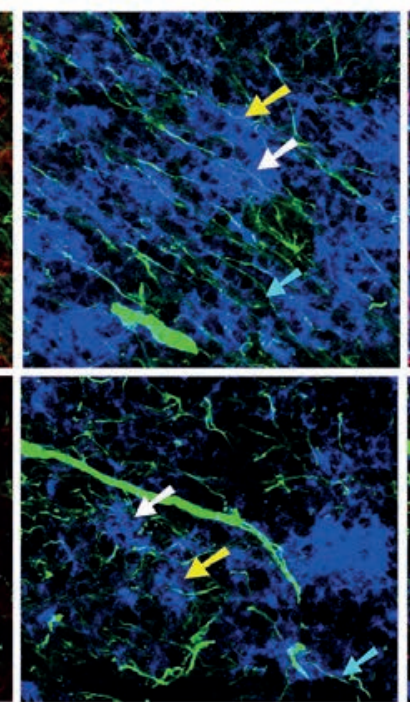

Merged

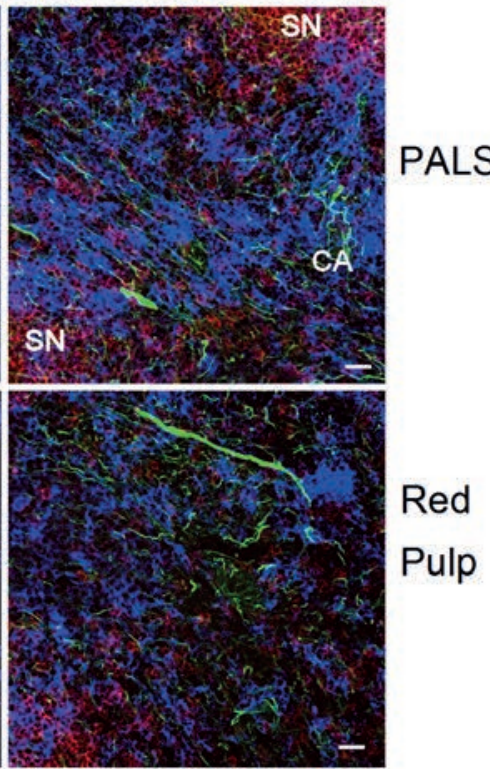

$\mathrm{B} 220+\mathrm{GFAP}+\mathrm{CD} 11 \mathrm{C}$

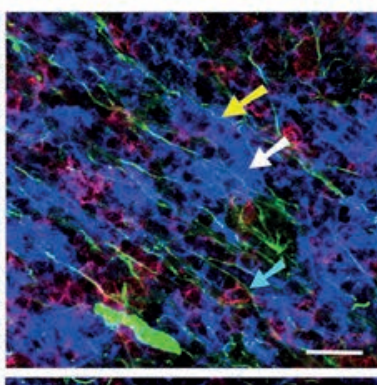

PALS

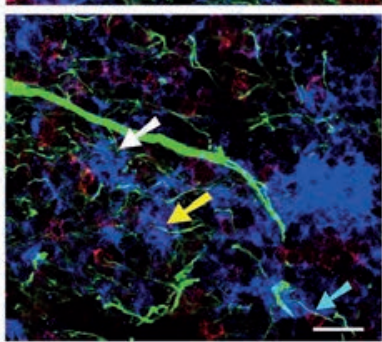

Red

Pulp

Figure 4. Distribution of NMSCs, B cells, and DCs in the PALS (A, C) and red pulp (B, D) of C57BL/6 mouse spleen. Antibodies against GFAP (green), B220 (red), and CD11c (blue) label mainly NMSCs, B cells, and DCs, respectively. C) High-resolution view of $(\AA)$ to show the interaction of NMSCs and immune cells in PALS. D) High-resolution view of (B) to show the interaction of NMSCs and immune cells in red pulp; cyan arrows indicate a $\mathrm{B}^{2} 20^{+} \mathrm{B}$ cell closely associated with NMSC processes; white arrows show a B220 $\mathrm{CD}_{11 \mathrm{c}^{+}}$DC closely apposed to NMSC processes; yellow arrows indicate a $\mathrm{B220}^{+} \mathrm{CD} 11 \mathrm{c}^{+} \mathrm{DC}$ with a close association with $\mathrm{NMSC}$ processes. CA, central artery; SN, splenic nodule; PALS, periarteriolar lymphoid sheath. Objective lens: 40x. Each image is a maximal intensity projection of a Z-stack. Stack size: $6.0 \mu \mathrm{m}$; optical slice interval: $0.50 \mu \mathrm{m}$. Scale bar: $20 \mu \mathrm{m}$. 
extensive network of NMSC processes in the red pulp (including the trabecula); these processes were very closely associated with

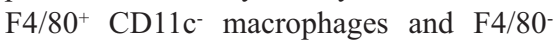

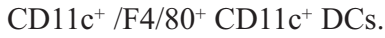

\section{Discussion}

In the present study, immunofluorescent staining and confocal microscopy/3D reconstruction were utilized to reveal the distribution of NMSCs and NMSC- leukocytes (lymphocytes, DCs, and macrophages) interactions in situ in the mouse spleen in order to gain a better understanding of the microanatomical basis of the bidirectional crosstalk of the PNS and secondary lymphoid tissue/organs. To the extent of our knowledge, we are the first to report these kinds of local interactions of NMSC and DCs/macrophages/lymphocytes in mouse spleen.

The spleen is organized into red pulp and white pulp, which are separated by an interface, namely the marginal zone. The white pulp of the spleen is structurally similar to a lymph node, contains $\mathrm{T}$ cell and $\mathrm{B}$ cell zones (the latter are also called the splenic nodule), and allows the generation of innate and adaptive immune responses that protect the body against blood-borne infections. ${ }^{14}$ By using an anti-GFAP antibody as a reliable cellular marker for NMSCs, we have analyzed the distribution of NMSCs in the spleen. We have previously investigated the distribution of NMSCs in mouse mesentery lymph nodes. ${ }^{29}$ Compared with this earlier study of mouse lymph node, we have made a few similar findings inside the splenic white pulp. First, an extensive network of NMSC/NMSC processes is present in mouse lymph node and spleen. Second, the distribution of NMSCs inside B cell regions (e.g., splenic nodules in spleen and B cell follicles in lymph node) is less extensive compared with that of T-cell dependent regions (PALS in spleen and paracortex in lymph node). Third, the NMSCs in both the lymph node and spleen are closely associated with blood vessels, indicating the possible neural control of blood flow inside the lymphoid organs.

As has been demonstrated in many studies and across a wide variety of species including human and mouse, the spleen is innervated by autonomic and sensory nerve fibers. ${ }^{9}, 10,15-20$ Noradrenergic postganglionic nerve fibers enter the spleen with the splenic artery, run along the trabeculae in plexuses, extend into the white pulp along
CD4

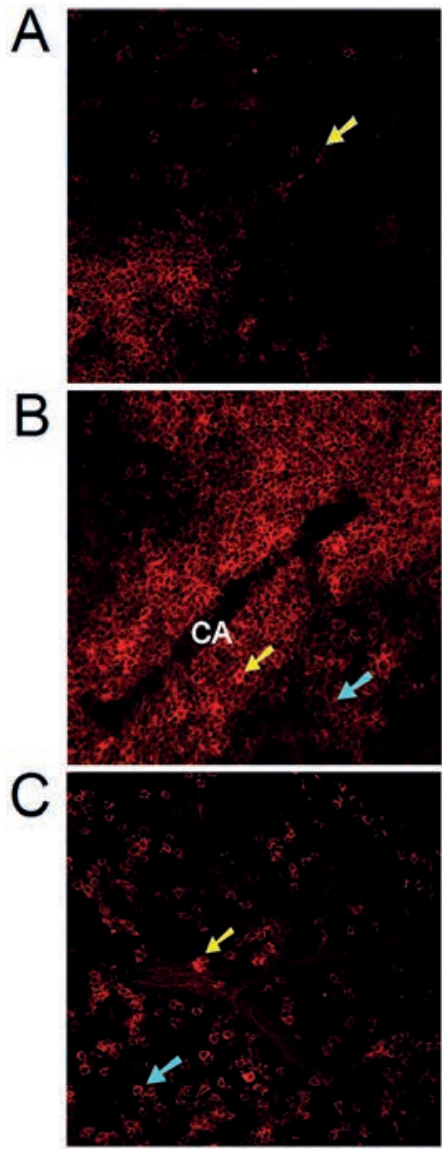

GFAP
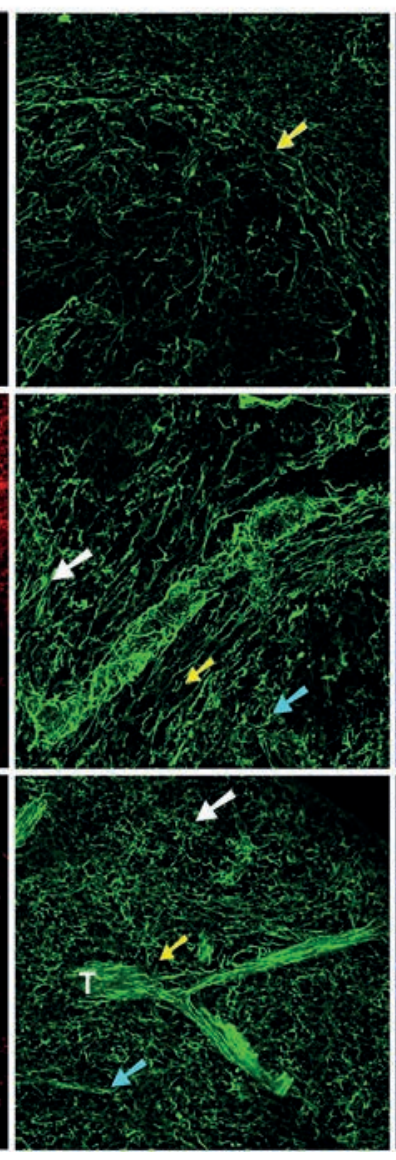

CD11c
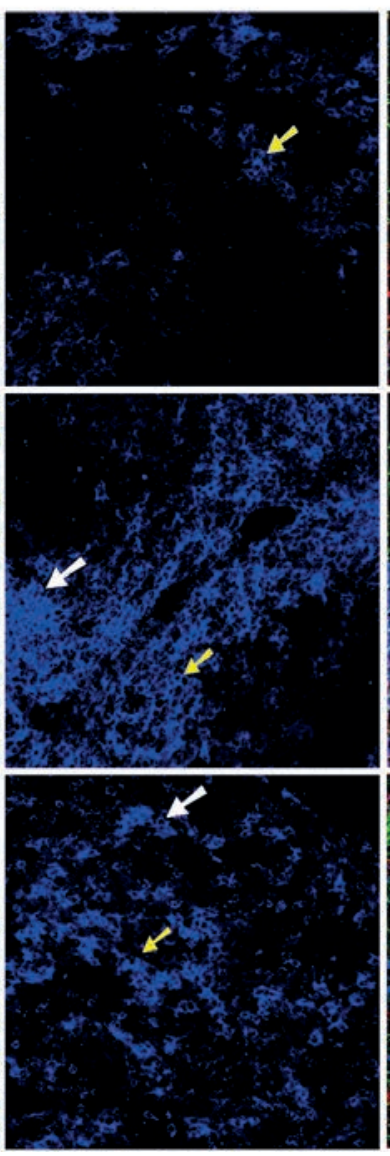

Merged

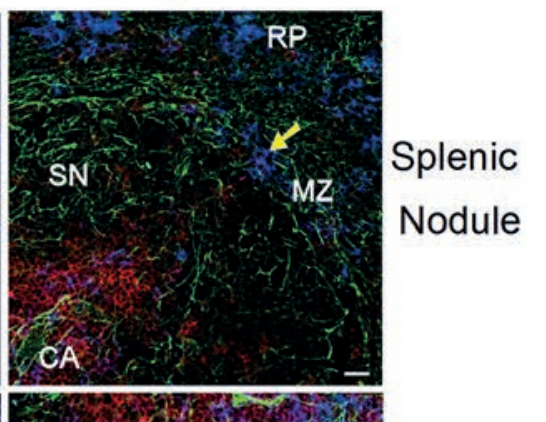

\section{PALS}

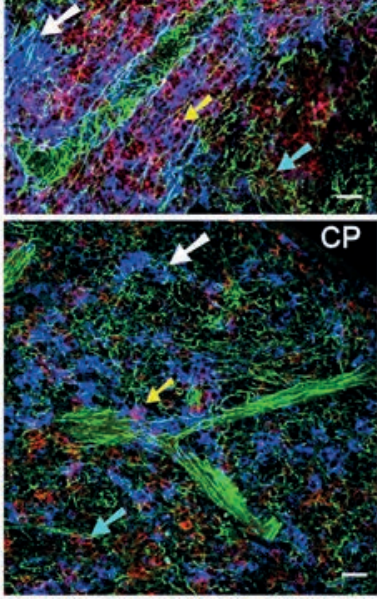

Red

Pulp

Figure 5. Distribution of NMSCs, T helper cells, and DCs in the splenic nodule (A), PALS (B), and red pulp (C) of C57BL/6 mouse spleen. Antibodies against GFAP (green), CD4 (red), and CD11c (blue) label mainly NMSCs, T helper cells, and DCs, respectively.The cyan arrows indicate a CD4+ T helper cell that is closely associated with NMSC process; white arrows show a CD4-CD11 ${ }^{+}$DC closely apposed to NMSC processes; yellow arrows indicate a $\mathrm{CD}^{+} \mathrm{CD} 11 \mathrm{c}^{+} \mathrm{DC}$ having a close association with NMSC processes; yellow arrows indicate a $\mathrm{CD} 4^{+} \mathrm{CD} 11 \mathrm{c}^{+} \mathrm{DC}$ having a close association with NMSC processes. MZ, marginal zone; CA, central artery; SN, splenic nodule; PALS, periarteriolar lymphoid sheath; CP, capsule; T, trabecula; RP, red pulp. Objective lens: 40x. Each image is a maximal intensity projection of a Z-stack. Stack size: $6.0 \mu \mathrm{m}$; optical slice interval: $0.50 \mu \mathrm{m}$. Scale bar: $20 \mu \mathrm{m}$. 
the central artery and end in the periarterial lymphatic sheath. ${ }^{9,19,31}$ Additionally, some studies have provided evidence for existence of the parasympathetic innervation of the spleen. ${ }^{9}$ However, the courses of the sympathetic nerves and parasympathetic nerves might be different. The sympathetic nerves may reach the spleen along with the arteries through the trabecula, whereas the parasympathetic nerves reach the spleen via both of its tips. ${ }^{9,16}$

Since NMSCs are essential components of Remak fibers, our study has demonstrated the presence of Remak nerve fibers in the mouse spleen indirectly. These Remak nerve fibers possibly include the Group C nerve fibers, the postganglionic sympathetic fibers, and some of the preganglionic sympathetic/parasympathetic fibers, and the motor nerve terminals at neuromuscular junctions..$^{22}$ In each compartment of spleen, including the splenic nodule, marginal zone, PALS, and red pulp, we have observed an extensive network of NMSC processes. Since NMSCs have been reported to be closely associated with nerve fibers including PGP9.5 $5^{+}$nerve fibers, tyrosine hydroxylase $(\mathrm{TH})^{+}$sympathetic nerve fibers, and neurofilament $(\mathrm{NF})^{+}$nerve fibers, ${ }^{32,33}$ the distribution of NMSCs as shown by our study also demonstrates the presence of nerve fibers, especially Remak nerve fibers, inside the mouse spleen.

Some previous reports ${ }^{9,19,31}$ have shown that noradrenergic innervation is particularly rich in T-cell zones and in areas of mast cells/macrophages, whereas the follicular and nodular zones in which B cells mature, are poorly innervated. Scattered nerve fibers have been observed in the red pulp primarily associated with plexuses along the trabeculae. However, our study has demonstrated the presence of a much denser innervation in both the white pulp (including PALS, marginal zones, and splenic nodule) and the red pulp of the spleen. We have also shown that these afferent nerve fibers are distributed through the trabecula and closely associated with blood vessels (including the central arteries and their branches, marginal sinuses, and splenic sinuses), indicating the neural control of blood flow inside the spleen. In our previous study of the lymph node, we observed this type of close association of NMSC and blood vessels (including high endothelial venules and other blood vessels). ${ }^{29}$ Therefore, the neuronal regulation of the blood flow and vascular permeability of blood vessels might affect the subsequent immune cell dynamics of the spleen.

Interconnections between the PNS and the immune system through dense innervations of secondary lymphoid tissue or organs have been reported, both anatomically and functionally, in some studies. $., 26,34,35$ Despite these published studies, detailed microanatomical studies of innervation of spleen and nerve-immune cell interactions remain rare. Therefore, in the present study, we have investigated the NMSC-immune cell interactions in situ inside the mouse spleen to address this lack.

Leukocytes in the spleen include various subsets of T/B cells, DCs, monocytes, neutrophils, and macrophages. ${ }^{14}$ Circulating $\mathrm{T}$ and $\mathrm{B}$ cells frequently enter secondary lymphoid organs searching for their cognate antigens. The trafficking and positioning of lymphocytes within defined splenic microenvironments enables the scanning of

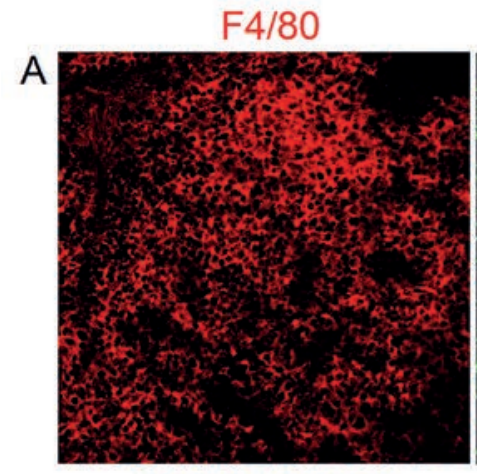

$\mathrm{F} 4 / 80+\mathrm{CD} 11 \mathrm{C}$

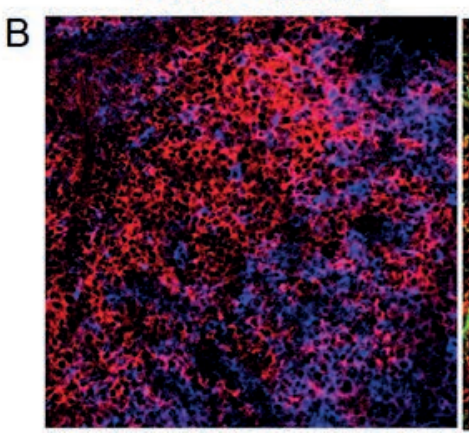

GFAP

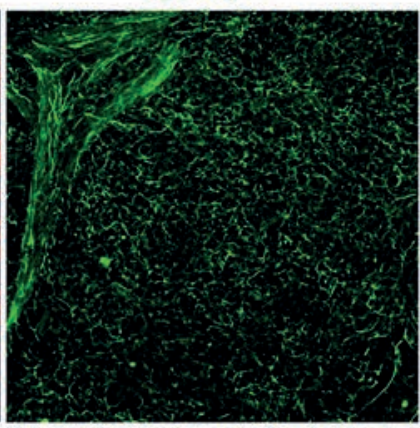

F4/80+GFAP

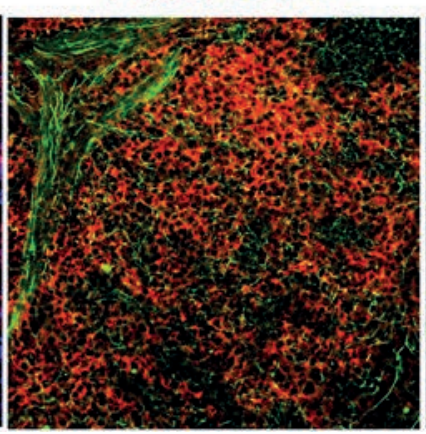

CD11c

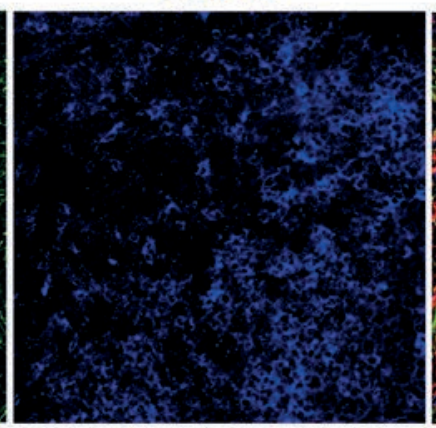

CD11C+ GFAP

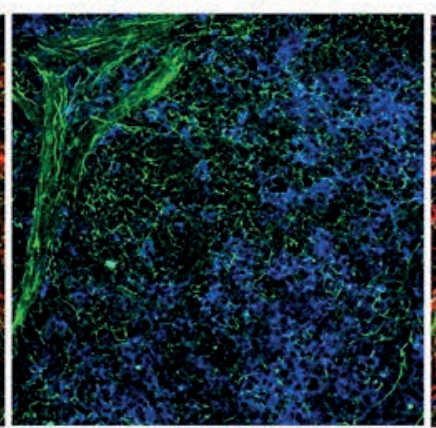

Merged

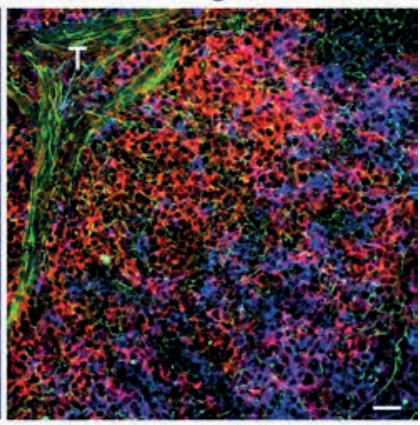

F4/80/ CD11C+GFAP

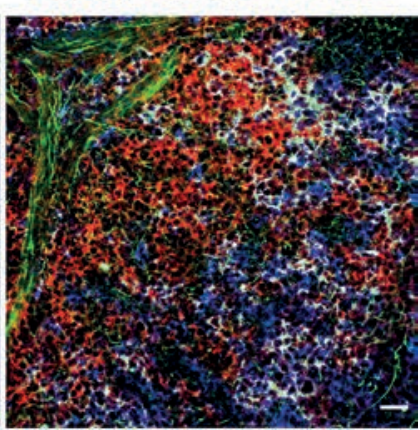

Figure 6. Interaction of NMSCs and macrophages in red pulp of spleen from C57BL/6 mouse. Antibodies against F4/80 (red), CD11c (blue), and GFAP (green) label mainly macrophages, DCs, and NMSCs in the red pulp, respectively. B) Various combinations of channels in (A) are shown here. In the fourth image from left, the $\mathrm{F} 4 / 80^{+} \mathrm{CD} 11 \mathrm{c}^{+} \mathrm{DCs}$ are marked in white after colocalization analysis. T, trabecula. Objective lens: 40x. Each image is a maximal intensity projection of a Z-stack. Stack size: $6.0 \mu \mathrm{m}$; optical slice interval: 0.50 $\mu \mathrm{m}$. Scale bar: $20 \mu \mathrm{m}$. 
antigen-presenting cells (APCs; including subsets of B cells) inside the spleen. ${ }^{14}$ The spleen contains distinct B cell lineages, including follicular and marginal zone B cells. Whereas follicular B cells recirculate and participate mainly in T-cell-dependent immune responses, marginal zone B cells reside between the marginal zone and red pulp, capture antigens in the blood via complement receptors, and promote both T cellindependent and -dependent immune responses. ${ }^{37,38}$ Several studies have reported the interaction of some B/T lymphocytes with $\mathrm{TH}^{+}$sympathetic norepinephrine nerve fibers in the splenic white pulp. ${ }^{19,36}$ However, no previous report has shown that the NMSCs interact with splenic lymphocytes in vivo. In our study, we have observed a very extensive interaction of NMSC with B cells (including follicular and marginal zone $\mathrm{B}$ cells) and $\mathrm{T}$ helper cells in the splenic nodule, PALS, marginal zone, and red pulp, indicating the neural regulation of the activities of immune cells.

The spleen hosts all major types of mononuclear phagocytes, including monocytes, macrophages, and DCs. These cells can protect the host because they identify pathogens and cellular stress, remove dead cells and foreign material, regulate tissue homeostasis and inflammatory responses, and initiate adaptive immunity. ${ }^{14,39}$ NerveDC interactions have been reported in various organs including the lung, lymph node, skin, and small intestine. ${ }^{34,40,41}$ Moreover, a few studies have reported the interaction of Schwann cells (including NMSCs) and DCs. ${ }^{29,41,42}$ In the present study, we have revealed extensive NMSC-DC interactions, as in our previous study. ${ }^{29}$ A few interesting findings should be mentioned concerning this type of interaction. First, around the central arteries and in the PALS, a cluster of DCs can be found, many of which have close associations with NMSC processes. Second, NMSCs interact with DCs in the splenic nodule and marginal DCs in the marginal zone of the splenic white pulp. Third, NMSCs are closely apposed to red pulp DCs. Fourth, the phenotypes of $\mathrm{DCs}^{43}$ associated with NMSCs included B220-

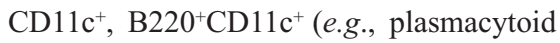
DCs) CD4-CD $11 \mathrm{c}^{+}$(lymphoid DCs) $\mathrm{CD}^{+} \mathrm{CD} 11 \mathrm{c}^{+}$(lymphoid DCs), F4/80$\mathrm{CD} 11 \mathrm{c}^{+}$, and $\mathrm{F} 4 / 80^{+} \mathrm{CD} 11 \mathrm{c}^{+}$in the white pulp and red pulp of the spleen. Although further studies of the molecular mechanism need to be carried out in vitro and in vivo, our findings provide a reliable microanatomical basis for the NMSC/nerve fibers to DC communications inside the spleen.

Splenic macrophages have two main protective activities during infections of blood-borne pathogens. The first and wellcharacterized is phagocytosis and the elimi- nation of pathogens from the circulation. ${ }^{44}$ In addition, splenic macrophages, which are better defined by the concomitant expression of F4/80, CD11b (at low levels), CD68, and other receptors, may play an important role in the activation of the immune system. ${ }^{44}$ Recent studies have also demonstrated the local interactions of macrophages and nerve fibers in the lung, lymph node, and other organs. ${ }^{15,34}$ However, the local interactions of macrophages and Schwann cells, which may support macrophage functions after peripheral nerve injury, have not yet been reported. In our study, we have observed that NMSCs have extensive and close appositions with $\mathrm{F} 4 / 80^{+} \mathrm{CD} 11 \mathrm{c}^{-}$macrophages in the red pulp, suggesting that NMSC/Remak fibers have effects on macrophage functions such as antigen presentation and cytokine production.

At the light microscopy level, the NMSC-immune cell contact has a "synapse-like" morphology, which has been described in our previous studies. ${ }^{29}$ Instead of being a random event described in some studies, ${ }^{10}$ this kind of cell-cell contact was very extensive inside the spleen, which formed the basis of direct synaptic neuroimmune communications. Since the NMSCs or nerve fibers are relatively static, and as immune cells such as DCs, macrophages, and lymphocytes might be quite mobile, this kind of contact/communication should be dynamic under health conditions and in diseases. In future studies, three approaches can be used to uncover the mechanisms of this type of cell-cell contact. The first is to confirm the interaction by using electron microscopy or immunoelectron microscopy. ${ }^{45}$ The second is to detect any direct ligand-receptor interactions. For example, some Schwann cells can express several ligands that are known to interact with macrophage receptors. ${ }^{46}$ The third is to identify any indirect contact/communications through neurotransmitters, cytokines, or other factors. ${ }^{47}$

In summary, our novel studies concerning splenic NMSC distribution and their interaction with leukocytes should provide new insights into the bidirectional crosstalk of secondary lymphoid tissue/organs and the PNS. Further in vivo and in vitro functional studies need to be performed to reveal the molecular mechanisms of these kinds of cell-cell communications of the PNS and immune system. ${ }^{1}$ The sympathetic and peptidergic innervation of spleen seems to have a role in the pathogenesis and progression of some neurologic, neuroimmune, and infectious/immune diseases. ${ }^{1,2}$ A better understanding of the interactions between the nervous and immune systems could accelerate the development of therapeutic strategies for diseases that involve altered neuroimmune cross-talk. ${ }^{2}$

\section{References}

1. Kenney MJ, Ganta CK. Autonomic nervous system and immune system interactions. Compr Physiol 2014;4:1177200.

2. Chavan SS, Pavlov VA, Tracey KJ. Mechanisms and therapeutic relevance of neuro-immune communication. Immunity 2017;46:927-42.

3. Tian L, Ma L, Kaarela T, Li Z. Neuroimmune crosstalk in the central nervous system and its significance for neurological diseases. J Neuroinflammation 2012;9:155.

4. Gabanyi I, Muller PA, Feighery L, Oliveira TY, Costa-Pinto FA, Mucida D. Neuro-immune interactions drive tissue programming in intestinal macrophages. Cell 2016;164:378-91.

5. Yoo BB, Mazmanian SK. The enteric network: Interactions between the immune and nervous systems of the gut. Immunity 2017;46:910-26.

6. Evans SS, Repasky EA, Fisher DT. Fever and the thermal regulation of immunity: the immune system feels the heat. Nat Rev Immunol 2015;15:33549.

7. Soto-Tinoco E, Guerrero-Vargas NN, Buijs RM. Interaction between the hypothalamus and the immune system. Exp Physiol 2016;101:1463-71.

8. ThyagaRajan S, Priyanka HP. Bidirectional communication between the neuroendocrine system and the immune system: relevance to health and diseases. Ann Neurosci 2012;19:40-6.

9. Jung WC, Levesque JP, Ruitenberg MJ. It takes nerve to fight back: The significance of neural innervation of the bone marrow and spleen for immune function. Semin Cell Dev Biol 2017;61:6070.

10. Murray K, Godinez DR, Brust-Mascher I, Miller EN, Gareau MG, Reardon C. Neuroanatomy of the spleen: Mapping the relationship between sympathetic neurons and lymphocytes. PLoS One 2017;12:e0182416.

11. Pacheco R, Contreras F, Prado C. Cells, molecules and mechanisms involved in the neuro-immune interaction. In: S. Gowder, editor. Cell interaction. InTech 2012. doi:10.5772/48435.

12. Takayanagi Y, Osawa S, Ikuma M, Takagaki K, Zhang J, Hamaya Y, et al. Norepinephrine suppresses IFN- $\gamma$ and TNF- $\alpha$ production by murine intestinal intraepithelial lymphocytes via the $\beta_{1}$ adrenoceptor. J Neuroimmonol 2012; 
245:66-74.

13. O'Neill HC, Griffiths KL, Periasamy P, Hinton RA, Hey YY, Petvises S, et al. Spleen as a site for hematopoiesis of a distinct antigen presenting cell type. Stem Cells Int 2011;2011:954275.

14. Bronte V, Pittet MJ. The spleen in local and systemic regulation of immunity. Immunity 2013;39:806-18.

15. Rosas-Ballina M, Ochani M, Parrish WR, Ochani K, Harris YT, Huston JM, et al. Splenic nerve is required for cholinergic antiinflammatory pathway control of TNF in endotoxemia. Proc Natl Acad Sci U S A 2008;105:1100813.

16. Buijs RM, van der Vliet J, Garidou ML, Huitinga I, Escobar C. Spleen vagal denervation inhibits the production of antibodies to circulating antigens. PLoS One 2008;3:e3152.

17. Anagnostou VK, DoussisAnagnostopoulou I, Tiniakos DG, Karandrea D, Agapitos E, Karakitsos P, et al. Ontogeny of intrinsic innervation in the human thymus and spleen. J Histochem Cytochem 2007;55:813-20.

18. Carlson SL, Albers KM, Beiting DJ, Parish M, Conner JM, Davis BM. NGF modulates sympathetic innervation of lymphoid tissues. J Neurosci 1995; 15:5892-9.

19. Felten DL, Ackerman KD, Wiegand SJ, Felten SY. Noradrenergic sympathetic innervation of the spleen: I. Nerve fibers associate with lymphocytes and macrophages in specific compartments of the splenic white pulp. J Neurosci Res 1987;18:28-36.

20. Nance DM, Sanders VM. Autonomic innervation and regulation of the immune system (1987-2007). Brain Behav Immun 2007;21:736-45.

21. Nave KA, Werner HB. Myelination of the nervous system: mechanisms and functions. Annu Rev Cell Dev Biol 2014;30:503-33.

22. Griffin JW, Thompson WJ. Biology and pathology of nonmyelinating Schwann cells. Glia 2008; 56:1518-31.

23. Kidd GJ, Ohno N, Trapp BD. Biology of Schwann cells. Handb Clin Neurol 2013;115:55-79.

24. Ydens E, Lornet G, Smits V, Goethals $\mathrm{S}$, Timmerman V, Janssens S. The neuroinflammatory role of Schwann cells in disease. Neurobiol Dis 2013; 5:95103.

25. Meyer zu Hörste G, Heidenreich H, Lehmann HC, Ferrone S, Hartung HP, Wiendl H, et al. Expression of antigen processing and presenting molecules by Schwann cells in inflammatory neuropathies. Glia 2010; 58:80-92.

26. Tzekova N, Heinen A, Küry P. Molecules involved in the crosstalk between immune- and peripheral nerve Schwann cells. J Clin Immunol 2014;34:S86-104.

27. Martini R, Fischer S, López-Vales R, David S. Interactions between Schwann cells and macrophages in injury and inherited demyelinating disease. Glia 2008;56:1566-77.

28. Im JS, Tapinos N, Chae GT, Illarionov PA, Besra GS, DeVries GH, et al. Expression of CD1d molecules by human schwann cells and potential interactions with immunoregulatory invariant $\mathrm{NK} \mathrm{T}$ cells. J Immunol 2006;177:5226-35.

29. Shi Z, Greene WK, Nicholls PK, Hu D, Tirnitz-Parker JEE, Yuan Q, et al. Immunofluorescent characterization of non-myelinating Schwann cells and their interactions with immune cells in mouse mesenteric lymph node. Eur J Histochem 2017;61:2827.

30. Owen GR, Häkkinen L, Wu C, Larjava $H$. A reproducible technique for specific labeling of antigens using preformed fluorescent molecular IgG-F(ab')2 complexes from primary antibodies of the same species. Microsc Res Tech 2010;73:623-30.

31. Perez SD, Silva D, Millar AB, Molinaro CA, Carter J, Bassett $\mathrm{K}$, et al. Sympathetic innervation of the spleen in male Brown Norway rats: a longitudinal aging study. Brain Res 2009; 1302:106-17.

32. Yamazaki S, Ema H, Karlsson G, Yamaguchi T, Miyoshi H, Shioda S, et al. Nonmyelinating Schwann cells maintain hematopoietic stem cell hibernation in the bone marrow niche. Cell 2011; 147:1146-58.

33. Defaweux V, Dorban G, Demonceau C, Piret J, Jolois $\mathrm{O}$, Thellin $\mathrm{O}$, et al. Interfaces between dendritic cells, other immune cells, and nerve fibres in mouse Peyer's patches: potential sites for neuroinvasion in prion diseases. Microsc Res Tech 2005;66:1-9.

34. Wülfing C, Günther HS. Dendritic cells and macrophages neurally hard-wired in the lymph node. Sci Rep 2015; 5:16866.

35. Huang J, Zhu C, Zhang P, Zhu Q, Liu Y, Zhu Z, et al. S100+ cells: a new neuroimmune cross-talkers in lymph organs. Sci Rep 2013;3:1114.
36. Gautron L, Rutkowski JM, Burton MD, Wei W, Wan Y, Elmquist JK. Neuronal and nonneuronal cholinergic structures in the mouse gastrointestinal tract and spleen. J Comp Neurol 2013;521:374167.

37. Tarlinton D, Good-Jacobson K. Diversity among memory B cells: origin, consequences, and utility. Science 2013;341:1205-11.

38. Pillai S, Cariappa A. The follicular versus marginal zone B lymphocyte cell fate decision. Nat Rev Immunol 2009; 9:767-77.

39. Kushwah R, Hu J. Complexity of dendritic cell subsets and their function in the host immune system. Immunology 2011;133:409-19.

40. Veres TZ, Shevchenko M, Krasteva G, Spies E, Prenzler F, Rochlitzer S, et al. Dendritic cell-nerve clusters are sites of $\mathrm{T}$ cell proliferation in allergic airway inflammation. Am J Pathol 2009;174: 808-17.

41. Riol-Blanco L, Ordovas-Montanes J, Perro M, Naval E, Thiriot A, Alvarez D, et al. Nociceptive sensory neurons drive interleukin-23-mediated psoriasiform skin inflammation. Nature 2014; 510:157-61.

42. Meyer zu Hörste G, Hu W, Hartung HP, Lehmann HC, Kieseier BC. The immunocompetence of Schwann cells. Muscle Nerve 2008; 37:3-13.

43. Hashimoto D, Miller J, Merad M. Dendritic cell and macrophage heterogeneity in vivo. Immunity 2011;35:32335.

44. Borges da Silva H, Fonseca R, Pereira RM, Cassado Ados A, Álvarez JM, D'Império Lima MR. Splenic macrophage subsets and their function during blood-borne infections. Front Immunol 2015;6:480.

45. Keith IM, Jin J, Saban R. Nerve-mast cell interaction in normal guinea pig urinary bladder. J Comp Neurol 1995;363:28-36.

46. Stratton JA, Shah PT. Macrophage polarization in nerve injury: do Schwann cells play a role? Neural Regen Res 2016;11:53-7.

47. Im JS, Tapinos N, Chae GT, Illarionov PA, Besra GS, DeVries GH, et al. Expression of CD1d molecules by human schwann cells and potential interactions with immunoregulatory invariant $\mathrm{NK} \mathrm{T}$ cells. J Immunol 2006;177:5226-35. 